\title{
Ultraschall in der Presse - die Medienarbeit der DEGUM
}

„Kann der Fötus die Ultraschallwelle hören und die Hitze spüren?“. Die Frage, was Ungeborene im Mutterleib wahrnehmen, beschäftigt nicht nur Experten und Leser der UiM (UiM 03/2012). Werdende Mütter, Väter, ja, die Öffentlichkeit im Allgemeinen ist daran interessiert, was ausgewiesene Experten zur allerersten Rundum-Untersuchung zu sagen haben. Die Pressestelle der DEGUM hat das Thema daher in 2012 aufgegriffen und für die Laien- und Fachpresse aufbereitet. Ungeborene Kinder, so die Kernaussage der DEGUM-Experten, tragen durch die Untersuchung keine gesundheitlichen Risiken. Leser zahlreicher Medien, überregional wie lokal, erfuhren so, dass werdende Mütter unbesorgt zur Schwangerschaftsuntersuchung gehen können. Beiträge darüber fanden sich unter anderem in der „Frankfurter Rundschau“, „Schweriner Volkszeitung“ oder „Bild der Frau“.

So breit wie das Spektrum der Ultraschallanwendungen so breit ist das Interesse der Öffentlichkeit an den Themen der DEGUM. Dies zeigte auch eine Pressekonferenz der DEGUM im Juni 2012 mit dem Titel „Ob Leber- oder Nierenkrebs, Gefäßriss oder Notfall - Kontrastmittelultraschall verbessert die Diagnostik“. Vertreter der Publikums- und Fachpresse nutzten interessante Ansatzpunkte, ihren Lesern und Hörern die vielfältigen Einsatzmöglichkeiten des Kontrastmittelultraschalls näher zu bringen. So berichtete etwa das „Deutschlandradio“ im Interview mit einem DEGUM-Experten über die durch CEUS verbesserte Beurteilung von Veränderungen an Leber und Niere. Die „Ärzte Zeitung“ betrachtete die Methode als Konkurrenz für CT und MRT: „Ob stumpfes Bauchtrauma, Flankenschmerz oder Bauchaorten-Aneurysma: Ein neues Verfahren aus der Welt des Ultraschalls mischt heute überall mit - und könnte in manchen Fällen CT und MRT überflüssig machen".

Die Pressestelle transportierte auch im Jahr 2012 neben neuesten wissenschaftlichen Aspekten auch bedeutende gesundheitspolitische Standpunkte der Fachgesellschaft in die Öffentlichkeit: „Die Ultraschalldiagnostik muss in der Hand des
Pressestelle kontinuierlich relevante Ultraschall-Themen auf.

Zur professionellen öffentlichen Präsenz einer Institution trägt maßgeblich deren Internetseite bei. Für eine gute Außenwirkung ist eine lebendige und gut gepflegte Homepage unerlässlich. Die DEGUM legt viel Wert auf ihre Online-Präsenz und nutzt diese vielseitig, denn sie dient nicht ausschließlich dem Werben um neue Mitglieder. Ziel ist vor allem auch der Austausch in den Sektionen und Arbeitskreisen innerhalb der DEGUM aber auch über aktuelle Ultraschallthemen interdisziplinär und mit verschiedenen Zielgruppen. Die Homepage macht die Arbeit der DEGUM für die Öffentlichkeit transparent und liefert sogar Patienten hilfreiche Informationen und Kontakte zu DEGUMzertifizierten Ärzten. Auch den Medien bietet die DEGUM hier einen eigenen Bereich, um sich über die Arbeit der Fachgesellschaft sowie aktuelle Forschungsergebnisse aus der Ultraschallmedizin zu informieren. Die Pressestelle pflegt permanent und in enger Abstimmung mit Vorstand und Geschäftsstelle neue Inhalte in die Homepage ein. Sie erarbeitet und aktualisiert Texte, wählt Bilder aus und stellt diese ein. Ein Medienspiegel auf der Homepage präsentiert eine Auswahl von aktuellen Online-Beiträgen diverser Fachund Publikumsmedien, die Themen der DEGUM aufgegriffen haben.

Die DEGUM-Pressestelle betreut die Homepage im Rahmen der gesamten Medienarbeit und nutzt dafür auch die Aktivitäten der DEGUM-Sektionen und -Arbeitskreise. Denn sie verfügen über fundierte Kenntnisse in ihrem Fachgebiet und sind auf dem neuesten Stand über aktuelle Entwicklungen, relevante Veranstaltungen und laufende Projekte. Die Pressestelle ist deshalb dankbar für Ihre Anregungen und Vorschläge zu Themen und freut sich über Ihre Unterstützung. Sprechen Sie uns bitte jederzeit an!

Kontakt: Deutsche Gesellschaft für Ultraschall in der Medizin (DEGUM)

Pressestelle: Irina Lorenz-Meyer/Anna Julia Voormann

Homepage: Christina Seddig/Julia Hommrich

Postfach 301120

70451 Stuttgart

Tel.: 0711/8931-642

Fax: 0711/8931-984

E-Mail: lorenz-meyer-@-medizinkommunikation.org 